\title{
Perspective
}

\section{Intubated, Awake, and Paralysed: A Never Event}

\author{
Huw Mayberry1, ${ }^{1}$, Alyssa M. Burgart2, ${ }^{2}$, Constantinos Kanaris ${ }^{3, *}$, (D) \\ ${ }^{1}$ Department of Paediatric Intensive Care, Alder Hey Children's NHS Foundation Trust, Liverpool, UK \\ ${ }^{2}$ Department of Anesthesiology, Stanford University School of Medicine, CA, US \\ ${ }^{3}$ Department of Paediatric Intensive Care, Cambridge University Hospitals NHS Foundation Trust, Cambridge, UK
}

\section{ARTICLE INFO \\ Article History \\ Received 06 August 2021 \\ Accepted 27 October 2021 \\ Keywords \\ Intubation \\ paralysis only intubation awareness during resuscitation \\ awareness during intubation \\ awareness during CPR \\ awareness during anaesthesia \\ critical care \\ intensive care}

\begin{abstract}
In this paper, we argue that paralysis-only intubation is almost never acceptable practice. We look at the evidence suggesting that this practice remains commonplace worldwide, its frequency has been exacerbated further by anaesthetic drug shortages secondary to the COVID-19 pandemic. We make a strong case that intubating a patient without sedation has such profound psychological and physiological risks that the practice is unethical and should be banned from medical practice with the exception of two clinical settings. These exceptions include (a) newborn intubation immediately after birth if there is immediate risk to life and (b) awake fibreoptic intubation whereby the patient has consented in advance, co-operative, and the airway has been prepared with local anaesthesia. We further argue that even in cases of cardiopulmonary resuscitation the practice impacts both on patient safety and clinician moral distress and as such in well-resourced times, there can be no justification for harming patients in this way. Formally labelling this practice as a Never Event is a way forward to homogeneously address the risks this archaic practice poses, both to our patients and to ourselves.
\end{abstract}

() 2021 First Affiliated Hospital of Zhengzhou University. Publishing services by Atlantis Press International B.V. This is an open access article distributed under the CC BY-NC 4.0 license (http://creativecommons.org/licenses/by-nc/4.0/).

\section{INTRODUCTION}

The practices of awake laryngoscopy without sedation and paralysis without sedation are archaic and cause patient harm. The purposeful withholding of sedation during paralysis must be consigned to the depths of clinical practice and listed as never events.

Paralysis-only intubation (the practice whereby a paralysing agent is administered without anaesthetic, allowing the patient to maintain awareness during the clinical process) is almost never acceptable practice. Even in Cardiopulmonary Resuscitation (CPR), evidence suggests this practice decreases patient safety. Muscle relaxation ablates the patient's ability to purposefully move, an important indicator of possible consciousness. Yet, despite the significant physiologic and psychological risks such practice poses to patients, literature suggests that this practice persists throughout the world. Crisis conditions in Brazil during the worst of the COVID-19 pandemic has led to a severe shortage of sedatives, with patients reportedly being intubated by being tied to the bed or with the use of neuromuscular blockade without sedation [1]. With no sedation available, clinicians have been forced to either torture their conscious patients, or allow them to die due to lack of ventilation. The documented patient harm and clinician moral distress from this disaster demonstrates that in well-resourced times, there can be no justification for harming patients in this way.

"Corresponding author.Email: ckanaris@doctors.net.uk

Peer review under responsibility of the First Affiliated Hospital of Zhengzhou University
Our recommendation excludes two important scenarios: (1) Intubation in the immediate postnatal period for newborns with airway compromise/an immediate threat to life/born in cardiac arrest where time taken to site a line may lead to preventable neurological damage. (2) Awake fibreoptic intubation where the patient is consented, co-operative and the airway is prepared with local anaesthesia.

\section{LEARNING FROM DREW HUGHES' DEATH}

A 13-year-old boy, Drew Hughes [2,3], spent the final moments of his life awake and paralysed as he became increasingly hypoxic from a misplaced Endotracheal (ET) tube in the back of an ambulance. Drew had suffered a head injury whilst skateboarding. A Computed Tomograph (CT) scan demonstrated a possible basilar skull fracture. His parents requested transfer to another hospital, and Drew was electively intubated as a precautionary measure for transport. Shortly after his intubation in the emergency department, Drew's sedation and paralysis wore off and he self-extubated. Drew was re-intubated and during transport, sat up on the stretcher and again self-extubated. He was aware enough to grab the arm of the paramedic and try to prevent them from giving vecuronium, before biting the finger of the Respiratory Therapist holding the endotracheal tube. He was given more paralytic medication without sedation, and the subsequent intubation was unsuccessful. The unrecognized oesophageal intubation led to Drew's cardiac arrest and death. This harrowing account highlights why giving adequate 
sedation alongside any paralysing agent during intubation is critical for patient safety. Had Drew been properly sedated for intubation and transportation, this tragic chain of events may have been prevented. Primum non nocere (First of all, do no harm).

\section{SCOPE OF THE PROBLEM}

Despite the known physiologic and psychological complications of intraoperative awareness, paralysis-only and inadequately sedated intubation remains in practice worldwide.

The literature shows widespread use of inadequate sedation or paralysis-only intubation in all age groups and in multiple parts of the world. In adults, Weingart et al. [4] found that less than half of 1.07 million patients undergoing endotracheal intubation in American emergency departments between 2006 and 2009 received sedative drugs. The 2021 ED-AWARENESS Study [5] found that $2.6 \%$ of ventilated patients in their cohort experienced awareness in the emergency department. Chong et al. [6] found that despite implementation of a sedation guideline in their hospital, $18 \%$ of their patients received a long acting paralytic without concurrent sedation.

In children, a 2012 case series in Pakistan showed that $42 \%$ of children were intubated without any medications and $2 \%$ were intubated with paralysis-only in the emergency department [7]. In 1998, Cook-Sather et al. [8] in comparing awake versus rapid sequence induction for patients with pyloric stenosis concluded controversially that infants could be safely intubated either awake or after anaesthesia and neuromuscular blockade. This led to letters to the editor [9] of Anesthesia and Analgesia from one group who were "disappointed that....awake intubation is still regarded as routine practice" and another [10] who questions on what basis the authors recommended that "when experienced pediatric anesthesiologists are available, anesthetised and paralysed tracheal intubation is recommended for otherwise normal infants with pyloric stenosis".

Awake intubation remains a prominent option in current difficult airway guidelines for paediatrics. Huang et al. [11] suggested in 2016 that the need for an awake intubation (defined by gastroesophageal reflux disease or airway obstruction at rest) should be ruled out before induction of anaesthesia whilst maintaining spontaneous ventilation. Raj et al. [12] recommended considering awake intubation alongside potential tracheostomy for those with a Colorado Paediatric Airway score of 16, indicating incompatibility with life without an artificial airway. The World Federation of Societies of Anaesthesiologists has recommended inhalational anaesthesia as their technique of choice for those with known difficult airways in their anaesthetic tutorial [13].

\section{PHYSIOLOGICAL SEQUELAE}

There are both physiological and psychological consequences to such actions.

Awake intubation in any age is associated with tachycardia or bradycardia, hypertension and oxygen desaturation [14,15]. Millar and Bissonaette [16] compared 13 neonates intubated either awake or with a thiopental and suxamethonium induction, demonstrating that intracranial pressure was increased in those who were intubated awake. Negative pressure pulmonary oedema has also been described as a potential hazard of muscle relaxants in awake infants. Warner et al. [17] described two infants who received vecuronium with inhalational anaesthesia. Oedema was attributed to paralysis of the muscles protecting the upper airway before there was significant depression of inspiratory muscle activity from the relaxant or inhalational agent. Intubation without sedation has been associated with poorer neurological outcomes in a study of 21,242 patients with trauma and Glasgow Coma Score (GCS) of less than 8 [18]. The prognosis of other groups of patients in this context remains unknown.

Topulos et al. [19] intubated four volunteers with only vecuronium and mechanically ventilated them. All described the process as extremely uncomfortable, three of the four requested pain relief at the end of the experiment and all four felt ill for 12-24 h afterward with feelings of muscle ache, fever, cough and sore throat. There were psychological sequelae including nightmares, anxiety, and preoccupation with death. Common sedatives, such as ketamine, midazolam, and propofol, are effective in preventing such awareness but the doses will depend on patient demographic (child vs adult) and clinical condition (reduced accordingly in cases of shock/cardiovascular instability).

During cardiac arrest, clinicians reasonably assume patients lack consciousness due to insufficient cardiac output. However, highly effective chest compressions may lead to regained consciousness, a phenomenon known as CPR Induced Consciousness (CPRIC), leading to physical manifestations of consciousness, such as eye opening and limb/trunk movements, speaking, etc. In a study from Australia [20] CPRIC was associated with a statistically significant increase in survival to hospital discharge. The use of vasodilatory sedatives (such as benzodiazepines and opioids) or muscle relaxants during resuscitation increased mortality. Studies are currently underway to determine if administering sedatives with more cardiovascularly stable profiles (like ketamine) during CPR will prevent CPRIC without the deleterious effects seen with other sedatives. While we cannot yet effectively prevent CPRIC during resuscitation, utilizing paralytic medications alone is deleterious to outcomes, and places patients at greater risk for preventable psychological sequelae than consciousness alone.

\section{PSYCHOLOGICAL SEQUELAE}

The NAP 5 project [21] from the Royal College of Anaesthetists in the UK investigated anaesthetic awareness, noting that accidental awareness during general anaesthesia can lead to post-traumatic stress disorder. Distress from awareness was most common when the patients had experienced paralysis alone or pain and paralysis together. Two patients believed that they were actually dead at the time of the intraoperative awareness due to experiencing paralysis. About $79 \%$ of patients who experienced distress had a moderate severe long-term impact on their psychological health. In the summary of the NAP 5 report, it concluded that "catastrophic interpretations of awareness experiences ... were strongly associated with serious longer-term sequelae. An experience of paralysis was important in this respect - more so than pain - in causing patient distress." The American Society of Anesthesiologists' Anesthesia Awareness Registry reports patient experiences of fear, anxiety, suffocation, sense of doom, sense of impending death, or similar reports that indicated emotional distress whilst experiencing awareness during anaesthesia. In their cohort, thirteen patients (20\%) reported that their experience was denied, or that they were blamed [22]. 
Studies by Blussé van Oud-Alblas et al. [23], Malviya et al. [24], and Lopez et al. [25] did not find significant psychological sequelae among children. However, in the Wake Up Safe dataset, Lee et al. [26] report, 11 of 14 cases of self-reported intra-operative awareness experienced harm, with five of those patients requiring the attention of a psychiatrist or psychologist.

In patients experiencing cardiac arrest, CPRIC is an increasingly recognised harmful phenomenon [16,27]. Data remains rare [28], but available data indicate around $40 \%$ of survivors of CPR recall events which occurred. In 2014, a prospective trial (AWARE) [29] interviewed 140 survivors of cardiac arrest finding that memories had one of seven themes: fear, animals/ plants, bright light, violence, déjà vu and family. About $2 \%$ could explicitly recall seeing and hearing actual events which took place during the resuscitative effort. AWARE 2 recruitment is currently underway [30] looking at whether patients can recall specific noises and images played to them during cardiac arrest. Further case reports have led to calls in some areas for consideration of sedation protocols for those in cardiac arrests [31,32]. If patients experience events during CPR, NAP 5 would suggest that induced paralysis would make this experience worse, whilst muscle relaxants are unlikely to be of benefit in patients with no airway reflexes. For non-survivors of CPR who are conscious, we worry their last experiences of consciousness are painful and traumatizing.

There are also historical accounts of children being ventilated with paralysing agents alone and no sedation for conditions like tetanus. Accounts of children subjected to this brutal practice provide insight into the trauma. "The boy had periods of emotional outbursts after coming off the ventilator during which he cried often and showed signs of frustration ..." [33]. Thankfully, medical practice has moved on from looking after ventilated patients using paralysis alone so there is no long-term outcome data on patients that have received long-term paralysis-only ventilation.

The discovery that paralysis agents had neither sedative nor analgesic qualities was first discovered by Dr Scott Smith, a resident who received d-turbocurarine under monitoring by colleagues [34], leading to change in common anaesthesia practice. Some physicians describe their battle with the ethics of subjecting patients to paralysis-only intubation. A few of these clinicians subjected themselves to paralysis-only intubation so that they could experience what patients have been going through [35]. These clinician accounts describe the immediate and lasting trauma experienced by having awareness during intubation, adding to the long list of evidence that paralysis only intubation is harmful to patients.

\section{WHY IS THIS COMPLICATION COMMON AND WHAT ARE THE BARRIERS TO QUALITY IMPROVEMENT?}

Literature data on inadequately sedated or paralysis-only intubation is scarce. On his blog "The Resus Room U.S." [36], Andrew Merelman suggests the reasons for poor sedation and analgesia of intubated patients include a misunderstanding of paralytic medications, a misunderstanding of the duration of action of induction agents, a fear of sedating critically ill or hemodynamically tenuous patients, and laziness.
Expert clinicians should have a firm grasp on the pharmacologic properties of the medications they administer. While we doubt clinician "laziness" is a primary reason for this practice, a lack of appreciation for the severe negative implications of awake paralysis may contribute. Fear of sedating haemodynamically tenuous patients is a genuine concern that may lead healthcare professionals to utilize paralytic medications without sedation. Administration of sedatives may lead to vasodilation and an expected drop in blood pressure. In very unstable patients such as those with sepsis and cardiomyopathy, administration of a standard dose of a sedative without administration of other agents to support blood pressure may precipitate severe hypotension, and even cardiac arrest.

In healthcare, especially emergency medicine, anaesthesia and intensive care, we regularly perform procedures in suboptimal conditions. Experts in these fields have the skill set to care for this population effectively and should use their considerable expertise to mitigate complications, including awareness. For example, pre-emptive use of vasoconstrictors such as noradrenaline and metaraminol may be utilised to offset the expectant vasodilation from sedation. The use of agents which result in minimal changes in blood pressure (such as ketamine) and volume expansion may also optimize conditions. If knowledge on this issue is lacking in pre-hospital settings, sharing of such knowledge should be prioritized to enhance excellence in pre-hospital care.

\section{A NEVER EVENT}

Never events refer to serious adverse events which must be reported. The event must be unambiguous, serious, and preventable, typically defined by patient death or serious harm. Such events require reporting so they may be analysed and help focus prevention efforts. Never Events described by the National Quality Forum in the USA include (amongst others) wrong site surgery and patient death or serious injury associated with a medication error [37]. National Health Service (NHS) Improvement in the UK lists transfusion of $\mathrm{ABO}$ incompatible blood products and misselection of high strength midazolam during conscious sedation amongst its Never Event list [38]. Australia (Sentinel Events) [39] and New Zealand (Always Review and Report Events) [40] have their own similar equivalents.

We believe intubation without sedation in conscious patients, paralysis-only intubation and leaving a patient on long-acting neuromuscular blockade without sedation should be added to each of these national lists.

These events are preventable through expert care. The NAP 5 audit demonstrates that awareness whilst paralysed can have a devastating psychological effect on patients. CPRIC data suggest paralytic medications during CPR decreases survival to discharge. Even participants who volunteered to be intubated with paralysis-only were left with anxiety, nightmares, and a preoccupation with death. Yet, these events continue to occur to this day.

\section{CONCLUSION}

The practice of intubation without sedation, paralysis-only intubation and long-acting neuromuscular blockade without concurrent 
sedation are harmful practices with potentially devastating consequences. The intervention to prevent such harm is simple and effective. When paralytic medication is clinically indicated, it takes minimal time to draw up an anaesthetic to complement paralysis. Intubating patients awake causes real harm; it is time to consign the practice to the past.

\section{CONFLICTS OF INTEREST}

The authors declare they have no conflicts of interest.

\section{AUTHORS' CONTRIBUTION}

All authors contributed equally to this paper.

\section{AUTHORS' NOTE}

Dedicated to Drew Hughes and his family, with gratitude for allowing us to use his story. We hope this is the first step in consigning paralysis only intubation to the past.

\section{REFERENCES}

[1] Alves L. Brazilian ICUs short of drugs and beds amid COVID-19 surge. Lancet 2021;397;1431-2.

[2] Dean B. Awake and paralyzed: the frightening final minutes for an injured teen. EMS 1 by Lexipol. 2016. Available from: https:// www.ems1.com/ems-products/medical-equipment/airway-management/articles/awake-and-paralyzed-the-frightening-finalminutes-for-an-injured-teen-yGRYgYgFXeqetlQG/ (accessed February 24, 2021).

[3] Hughes D. Patient story: Drew Hughes. Patient safety movement foundation. Available from: https://patientsafetymovement.org/ advocacy/patients-and-families/patient-stories/drew-hughes/ (accessed February 24, 2021).

[4] Weingart GS, Carlson JN, Callaway CW, Frank R, Wang HE. Estimates of sedation in patients undergoing endotracheal intubation in US EDs. Am J Emerg Med 2013;31;222-6.

[5] Pappal RD, Roberts BW, Mohr NM, Ablordeppey E, Wessman BT, Drewry AM, et al. The ED-AWARENESS study: a prospective, observational cohort study of awareness with paralysis in mechanically ventilated patients admitted from the emergency department. Ann Emerg Med 2021;77;532-44.

[6] Chong ID, Sandefur BJ, Rimmelin DE, Arbelaez C, Brown CA, Walls RM, et al. Long-acting neuromuscular paralysis without concurrent sedation in emergency care. Am J Emerg Med 2014;32;452-6.

[7] Bano S, Akhtar S, Zia N, Khan UR, Haq Au. Pediatric endotracheal intubations for airway management in the emergency department. Pediatr Emerg Care 2012;28;1129-31.

[8] Cook-Sather SD, Tulloch HV, Cnaan A, Nicolson SC, Cubina ML, Gallagher PR, et al. A comparison of awake versus paralyzed tracheal intubation for infants with pyloric stenosis. Anesth Analg $1998 ; 86 ; 945-51$.
[9] Theroux MC. Tracheal intubation for awake versus paralyzed infants with pyloric stenosis. Anesth Analg 1998;87;1455; author reply $1456-7$.

[10] Armstrong TS, Byers GF, Johnston GM. Tracheal intubation in infants. Anesth Analg 1998;87;1455-6.

[11] Huang AS, Rutland L, Hajduk J, Jagannathan N. Difficult airway management of children in ambulatory anesthesia: challenges and solutions. Ambul Anesth 2016;3;37-45.

[12] Raj D, Luginbuehl I. Managing the difficult airway in the syndromic child. Contin Educ Anaesth Crit Care Pain 2015;15;7-13.

[13] Prasad Y. The difficult pediatric airway anaesthesia tutorial of the week. World Federation of Societies of Anaesthesiologists. 2021. Available from: https://resources.wfsahq.org/atotw/the-difficultpaediatric-airway-anaesthesia-tutorial-of-the-week-250/ (accessed February 24, 2021).

[14] Xue FS, Zhang GH, Sun HY, Li CW, Li P, Sun HT, et al. Blood pressure and heart rate changes during intubation: a comparison of direct laryngoscopy and a fibreoptic method. Anaesthesia 2006;61;444-8.

[15] Xue FS, Zhang GH, Sun HT, Li CW, Liu KP, Xu YC, et al. Blood pressure and heart rate changes during fibreoptic orotracheal intubation: a comparison of children and adults. Eur J Anaesthesiol 2007;24;39-45.

[16] Millar C, Bissonnette B. Awake intubation increases intracranial pressure without affecting cerebral blood flow velocity in infants. Can J Anaesth 1994;41;281-7.

[17] Warner LO, Martino JD, Davidson PJ, Beach TP. Negative pressure pulmonary oedema: a potential hazard of muscle relaxants in awake infants. Can J Anaesth 1990;37;580-3.

[18] Hoffmann M, Czorlich P, Lehmann W, Spiro AS, Rueger JM, Lefering R. The impact of prehospital intubation with and without sedation on outcome in trauma patients with a GCS of 8 or less. J Neurosurg Anesthesiol 2017;29;161-7.

[19] Topulos GP, Lansing RW, Banzett RB. The experience of complete neuromuscular blockade in awake humans. J Clin Anesth 1993;5;369-74.

[20] Olaussen A, Nehme Z, Shepherd M, Jennings PA, Bernard S, Mitra B, et al. Consciousness induced during cardiopulmonary resuscitation: an observational study. Resuscitation 2017;113; $44-50$.

[21] Pandit JJ, Andrade J, Bogod DG, Hitchman JM, Jonker WR, Lucas N, et al. 5th National Audit Project (NAP5) on accidental awareness during general anaesthesia: summary of main findings and risk factors. Br J Anaesth 2014;113;549-59.

[22] Kent CD, Posner KL, Mashour GA, Mincer SL, Bruchas RR, Harvey AE, et al. Patient perspectives on intraoperative awareness with explicit recall: report from a North American anaesthesia awareness registry. Br J Anaesth 2015;115;I114-I21.

[23] Blussé van Oud-Alblas HJ, van Dijk M, Liu C, Tibboel D, Klein J, Weber F. Intraoperative awareness during paediatric anaesthesia. Br J Anaesth 2009;102;104-10.

[24] Malviya S, Galinkin JL, Bannister CF, Burke C, Zuk J, Popenhagen $\mathrm{M}$, et al. The incidence of intraoperative awareness in children: childhood awareness and recall evaluation. Anesth Analg 2009; 109;1421-7.

[25] Lopez U, Habre W, Van der Linden M, Iselin-Chaves IA. Intraoperative awareness in children and post-traumatic stress disorder. Anaesthesia 2008;63;474-81.

[26] Lee AC, Redding AT, Tjia I, Rana MS, Heitmiller E. Self-reported awareness during general anesthesia in pediatric patients: a study from Wake Up Safe. Paediatr Anaesth 2021;31;676-85. 
[27] Versteeg J, Noordergraaf J, Vis L, Willems P, Bremer R. CPRinduced consciousness: attention required for caregivers and medication. Resuscitation 2019;142;E35.

[28] Varon J. Awareness during resuscitation: Where is the data? Am J Emerg Med 2019;37;952-3.

[29] Parnia S, Spearpoint K, de Vos G, Fenwick P, Goldberg D, Yang J, et al. AWARE - AWAreness during REsuscitation - a prospective study. Resuscitation 2014;85;1799-805.

[30] NHSHealth Research Authority. AwareII.AWAREII(AWAreness during REsuscitation) - A Multi-Centre Observational Study of the Relationship between the quality of brain resuscitation and consciousness, neurological, functional and cognitive outcomes following cardiac arrest. Available from: https://www.hra.nhs. uk/planning-and-improving-research/application-summaries/research-summaries/aware-ii/ (accessed February 24, 2021).

[31] Sukumar V. Having a conscious patient during cardiopulmonary resuscitation: is it not time to consider sedation protocol?: a case report. A A Pract 2019;13;250-2.

[32] Rice DT, Nudell NG, Habrat DA, Smith JE, Ernest EV. CPR induced consciousness: sedation protocols for this special population. Br Paramed J 2016;1;24-30.

[33] Ramsey FC. Total paralysis without sedation in the treatment of severe tetanus. Dev Med Child Neurol 1965;7;56-60.
[34] Anderson R. A TORTURED PATH: curare's journey from poison darts to paralysis by design. Mol Interv 2010;10;252-8.

[35] Lyon Maj RF. Take my breath away. Ann Emerg Med 2017;69; 253-4.

[36] Merelman A. Paralysis with sedation - a negligent act. The Resus Room U.S. Available from: https://theresusroom.com/ 2018/11/30/paralysis-without-sedation-a-negligent-act/ (accessed February 24, 2021).

[37] National Quality Forum. List of serious reportable events (aka SRE or "Never Events"). Available from: https://www.qualityforum.org/Topics/SREs/List_of_SREs.aspx (accessed July 4, 2021).

[38] National Health Service. Revised never events policy and framework. Available from: https://www.england.nhs.uk/patientsafety/revised-never-events-policy-and-framework/ (accessed July 4, 2021).

[39] Australian Commission on Safety and Quality in Health Care. Australian sentinel events list. Available from: https://www. safetyandquality.gov.au/our-work/indicators/australiansentinel-events-list (accessed July 4, 2021).

[40] Health Quality and Safety Commission New Zealand. Always report and review list 2018-19. Available from: https://www. hqsc.govt.nz/assets/Reportable-Events/Publications/National_ Adverse_Events_Policy_2017/Always-report-and-review-list2018-19-Final.pdf (accessed July 4, 2021). 